\title{
LED-based Wake-up Circuit for Microcontrollers
}

\author{
E. Ripoll-Vercellone, F. Reverter, and M. Gasulla, Senior Member, IEEE
}

\begin{abstract}
This paper proposes, analyzes, and tests a wake-up circuit for a microcontroller (MCU) that uses a LED, operating as a photodetector, illuminated by a smartphone flashlight. The wake-up circuit consists of a high-pass filter and a voltage level translator that interfaces the LED, with a suitable resistor in parallel, to the MCU. When illuminated by a switching flashlight, the LED generates a square voltage that is conveniently converted in logic levels at the output of the wake-up circuit. A firmware embedded into the MCU additionally checks that a predetermined sequence of logic pulses at a given rate is accomplished to activate the MCU. The paper includes a theoretical analysis and experimental results that validate the proposed circuit.
\end{abstract}

Index Terms-Optical wake-up circuit, microcontroller, lowpower mode, LED, photodiode.

\section{INTRODUCTION}

Wireless sensor nodes usually are autonomously powered and have a microcontroller unit (MCU) that controls the electronic modules and processes the information coming from sensors and/or transceivers [1]. To save power, the sensor node is set most of the time in a low-power mode (LPM), which is controlled by the MCU, and only wakes-up occasionally for sensing and transmitting data. MCUs are awaken by internally and/or externally generated interruption signals. Internal interruption signals come from embedded timers and are used by the MCU to carry out periodic tasks [1], [2]. External interruption signals come from, among others, the same sensors in charge of the measurement [3], secondary low-power sensors [4], alarm events [5], or remote radiofrequency (RF) signals that trigger a wake-up receiver of the sensor node [6].

Optical wake-up has been proposed using detectors such as LDRs [4] and photodiodes [7], [8]. In [7], a commercial infrared photodiode was used with a transimpedance amplifier, active and passive filters, a comparator, and some additional circuitry. Undesired effects of artificial and ambient lights were prevented at the cost of circuit complexity. In [8], a custom CMOS receiver is proposed, where a parasitic photodiode is again surrounded of a complex circuitry. Contrariwise, in [4] a simple voltage divider was used. There, ambient light was the wake-up source and, hence, no circuitry was added to filter it out. However, a current of $3.3 \mu \mathrm{A}$ was continuously drained and, because no high-pass filter was used, generated voltages lying between a logic ' 0 ' and ' 1 ' could lead to an increase of the MCU power consumption [9]. LEDs can also be employed as optical detectors, as demonstrated in [10], and hence for low-cost optical wake-up circuits, as initially proven in [11]. There, a LED was directly connected to the MCU and a smartphone flashlight was used as the wake-up source. Besides, the LED was used for light signaling, thus having a dual

This paragraph of the first footnote will contain the date on which you submitted your brief for review. This work was supported by the Secretariat of University and Research of the Ministry of Business and Knowledge of the Government of Catalonia, by the Spanish State Research Agency (AEI) and by the European Regional Development Fund under Project TEC2016-76991-P. function. However, interfering lights, even of low intensity, could also wake-up the MCU and increase its power consumption, as in [4]. In addition, no detailed analysis is provided. Here, a LED-based wake-up circuit activated by a smartphone flashlight and with immunity to interfering lights is proposed, analyzed, and tested. The proposed circuit is much simpler than those suggested in [7], [8], and solves the limitations of interference sensitivity and current consumption found in [4], [11].

\section{WAKE-UP CIRCUIT}

The circuit of Fig. 1 is proposed for interfacing a LED to the MCU, which includes several stages as described throughout this section. Similarly to a photodiode [10], a voltage across the LED $\left(v_{\mathrm{D}}\right)$ is generated in presence of light, e.g. that coming from a smartphone flashlight. The value of $v_{\text {D }}$ needs to be high enough to become a logic ' 1 ' and wake-up the MCU. However, artificial and ambient interfering lights could also wake-up the MCU. To palliate this, a resistor $\left(R_{\mathrm{D}}\right)$ is added in parallel with the LED [12]. In this way, $v_{\mathrm{D}}$ is proportional to the generated LED current $\left(i_{\mathrm{D}}\right)$, thus allowing a better discrimination between different light intensities. Even so, DC and low frequency signals coming from interfering lights either indoors (e.g. office) or outdoors (e.g. sun) can generate values of $v_{\text {D }}$ corresponding to a (false) ' 1 ' or to an intermediate level between a logic ' 0 ' and ' 1 ' that leads to an increase of the MCU power consumption [9]. For this reason, a high-pass filter (HPF), consisting of $C_{\mathrm{H}}$ and $R_{\mathrm{H}}$, is added to suppress these signals. Consequently, a switching wake-up flashlight has to be used to surpass the HPF. When the flashlight turns on, $i_{\mathrm{D}}$ and thus $v_{\mathrm{D}}$ undergo a step increase from an initial value (corresponding to the interfering light) and the voltage step $\left(\Delta v_{\mathrm{D}}\right)$ instantaneously appears in the HPF output $\left(v_{\mathrm{H}}\right)$. Since $\Delta v_{\mathrm{D}}$ may not directly lead to a ' 1 ', an inverter voltage level translator (VLT) stage is also added at the output of the HPF. This stage, based on a NMOS transistor and a pull-up resistor $\left(R_{\mathrm{M}}\right)$, converts $\Delta v_{\mathrm{D}}$ at its input to $0 \mathrm{~V}\left(\mathrm{a}^{\prime}{ }^{\prime} 0\right.$ ') at its output $\left(v_{\mathrm{M}}\right)$ and a zero voltage to $V_{\text {cc }}\left(\mathrm{a}^{\prime}\right.$ ' 1 '). Finally, the VLT output is connected to an interruption pin of the MCU.

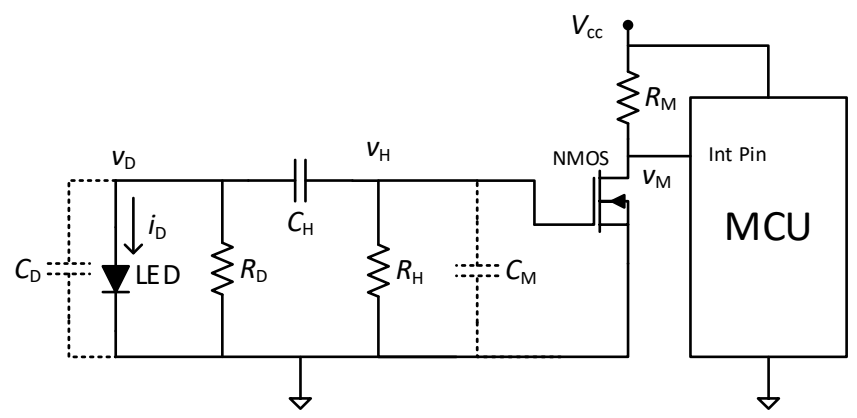

Fig. 1. Circuit for waking up a microcontroller using a LED.

E. Ripoll-Vercellone is with Idneo Technologies, 08100 Mollet del Vallès, Catalonia, Spain (e-mail: edgar.ripoll@idneo.com).

F. Reverter and M. Gasulla are with the Department of Electronic Engineering, Universitat Politècnica de Catalunya, 08860 Castelldefels, Catalonia, Spain (e-mails: ferran.reverter@upc.edu, manel.gasulla@upc.edu). 
In order to evaluate the response of the circuit of Fig. 1 in the frequency and time domains, the parasitic capacitances of the LED $\left(C_{\mathrm{D}}\right)$ and of the NMOS input $\left(C_{\mathrm{M}}\right)$ are considered and represented in dashed lines. In addition, the LED itself is considered for the analysis as a current source $\left(i_{\mathrm{D}}\right)$. To avoid loading effects on $R_{\mathrm{D}}$ and $C_{\mathrm{H}}$, the conditions $R_{\mathrm{H}} \gg>R_{\mathrm{D}}$ and $C_{\mathrm{D}}, C_{\mathrm{M}}<<C_{\mathrm{H}}$ are imposed. Thus, the Laplace transfer function of the circuit is given by

$H(s)=\frac{V_{\mathrm{H}}}{I_{\mathrm{D}}} \approx-R_{D} \frac{s \omega_{2}}{\left(s+\omega_{1}\right)\left(s+\omega_{2}\right)}$

which corresponds to a band-pass filter (BPF) with the following cutoff frequencies

$f_{1}=\frac{\omega_{1}}{2 \pi}=\frac{1}{2 \pi \tau_{1}}$

$f_{2}=\frac{\omega_{2}}{2 \pi}=\frac{1}{2 \pi \tau_{2}}$

with $\tau_{1}=R_{\mathrm{H}} C_{\mathrm{H}}$ and $\tau_{2}=R_{\mathrm{D}}\left(C_{\mathrm{D}}+C_{\mathrm{M}}\right)$, where $\tau_{1}>>\tau_{2}$ and thus $f_{1}<<f_{2}$.

A wake-up flashlight of frequency $f_{\mathrm{s}}$ will generate a square-wave signal of the same frequency for $i_{\mathrm{D}}$ and thus, assuming $f_{\mathrm{s}}<<f_{2}$, for $v_{\mathrm{D}}$. At a rising edge of $v_{\mathrm{D}}, v_{\mathrm{H}}$ sharply increases from zero to $\Delta v_{\mathrm{D}}$ and $v_{\mathrm{M}}$ toggles from ' 1 ' to ' 0 '. Then, $v_{\mathrm{H}}$ exponentially decreases to zero. When $v_{\mathrm{H}}$ crosses the threshold voltage of the $\operatorname{NMOS}\left(V_{\mathrm{T}}\right), v_{\mathrm{M}}$ toggles again to ' 1 '. This process repeats at each rising edge of $v_{\mathrm{D}}$. For this behavior, $v_{\mathrm{H}}$ is assumed nearly zero before the rising edges of $v_{\mathrm{D}}$, which can be achieved, assuming a square-wave flashlight of period $T_{\mathrm{s}}\left(=1 / f_{\mathrm{s}}\right)$ and a duty cycle of $50 \%$, with $0.5 T_{\mathrm{s}}>5 \tau_{1}$, leading to

$f_{1}>\frac{5}{\pi} f_{\mathrm{s}}$

A Firmware Activation Check (FAC) is embedded into the MCU to reject unwanted wake-up signals coming from interfering lights or even from hacking lights. On the first rising edge of $v_{\mathrm{M}}$, the MCU is awaken and the FAC is executed. If the result of the FAC is correct, the MCU is kept active; on the contrary, the MCU returns to LPM.

Changes in the parameter values of the components due to their tolerance or temperature drift should not affect the circuit performance thanks to its digital activation mechanism. A lower flashlight intensity or a change in $R_{\mathrm{D}}$ lowers $\Delta v_{\mathrm{D}}$ but only a value higher than $V_{\mathrm{T}}$ is required, which can also slightly change, altering the duty cycle of $v_{\mathrm{M}}$ but not its frequency. Changes on $R_{\mathrm{H}}$ and $C_{\mathrm{H}}$ alter $f_{1}$ but it is easy to accomplish (4) in the worst-case scenario. Finally, the two logic levels of $v_{\mathrm{M}}$ are not affected by voltage drifts on $V_{\mathrm{cc}}$ since it powers both the VLT and the MCU.

\section{EXPERIMENTAL RESULTS}

As a proof-of-concept, a prototype of the circuit in Fig. 1 was designed with the following devices: 1) a low-cost flat-top red LED (150060RS75000, Wurth Electronics) that provides high enough values of $\Delta v_{\mathrm{D}}$ at indoor and outdoor conditions without requiring very high values of $R_{\mathrm{D}}$ [12]; 2) a small-signal NMOS (RE1C002UN, RHOM) that offers low values of both $C_{\mathrm{M}}$ and $V_{\mathrm{T}}$; and 3 ) an STM32L072 MCU with an interruption pin (PA0) that is activated at the rising edge of $v_{\mathrm{M}}$. For illuminating the LED, we used, depending on the performed tests, either the flashlight of a Motorola Moto X Style (XT1572) smartphone placed at a distance of $1 \mathrm{~cm}$ or a high-power white LED that emulated it [12].

\section{A. Static Characterization}

Fig. 2 shows the static current/voltage (I/V) characterization of the LED with different types of lights. The Flash curve is the signal of interest, generated here with the emulated flashlight. The rest are interfering lights: Office curve corresponds to compact fluorescent lights placed on the ceiling ( 700 lux), Shadow curve to sunlight blocked by an object ( $\sim 60 \mathrm{~W} / \mathrm{m} 2)$, Sun curve to direct sunlight $(\sim 1000$ $\mathrm{W} / \mathrm{m} 2$ ). Office and Shadow curves offer the lowest current levels, and Flash curve a higher current level than Sun curve. The Sun+Flash curve is a combination of direct sunlight and flashlight and presents highest current level.

Three lines corresponding to the $\mathrm{I} / \mathrm{V}$ curves of three different values of $R_{\mathrm{D}}$ are also shown $\left(R_{\mathrm{D} 0}, R_{\mathrm{D} 1}\right.$, and $\left.R_{\mathrm{D} 2}\right)$. Operating values of $v_{\mathrm{D}}$ arise from the intersection of the LED curves with the resistor lines. $R_{\mathrm{D} 1}$ of $500 \mathrm{k} \Omega$ is selected for intersecting with the knee of the Flash curve, resulting in the values of $v_{\mathrm{D}}$ shown in Table I. This selection maximizes $\Delta v_{\text {D }}$, also shown in Table I, which refers to the difference between the value of $v_{\mathrm{D}}$ corresponding to the Flash curve and any of the interfering lights. Obviously, the worst case (minimum $\Delta v_{\mathrm{D}}$ ) is for the Sun case, but the resulting value of $0.62 \mathrm{~V}$ is still good enough to toggle the VLT output. Smaller (e.g. $R_{\mathrm{D} 2}$ ) or higher values of $R_{\mathrm{D}}$ (e.g. $\left.R_{\mathrm{D} 0}\right)$ lead to smaller values of $\Delta v_{\mathrm{D}}$. In particular, for $R_{\mathrm{D} 0}$ (infinite value, equivalent to using no $R_{\mathrm{D}}$ ) much smaller values of $\Delta v_{\mathrm{D}}$ are achieved (e.g. $60 \mathrm{mV}$ ), which are not high enough to toggle the VLT output. Hence, this demonstrates the need of using $R_{\mathrm{D}}$, as pointed out in Section II.

Actually, the flashlight will add to the interfering light, so the Flash curve should add the corresponding interfering curve. Adding Office or Shadow curves to Flash curve does not significantly change it and $R_{\mathrm{D} 1}$ is still a good choice. However, a significant change appears when adding the Sun curve resulting in the Sun+Flash curve. In this case, $R_{\mathrm{D} 2}$ of $340 \mathrm{k} \Omega$, which intersects with the knee of the Sun+Flash curve, could seem more optimum, since it leads to $\Delta v_{\mathrm{D}}=0.85 \mathrm{~V}$ (shown in Table I at the row of Sun+Flash) when changing from Sun to Sun + Flash. However, $\Delta v_{\mathrm{D}}$ decreases for the other interfering lights with respect to using $R_{\mathrm{D} 1}$. Furthermore, the smartphone, when placed near the MCU for activating it, will block any direct interfering light and thus the actual interfering light will be of low intensity, e.g. like in Shadow or Office (see Section III.C). Thus, $R_{\mathrm{D} 1}$ is still the best option.

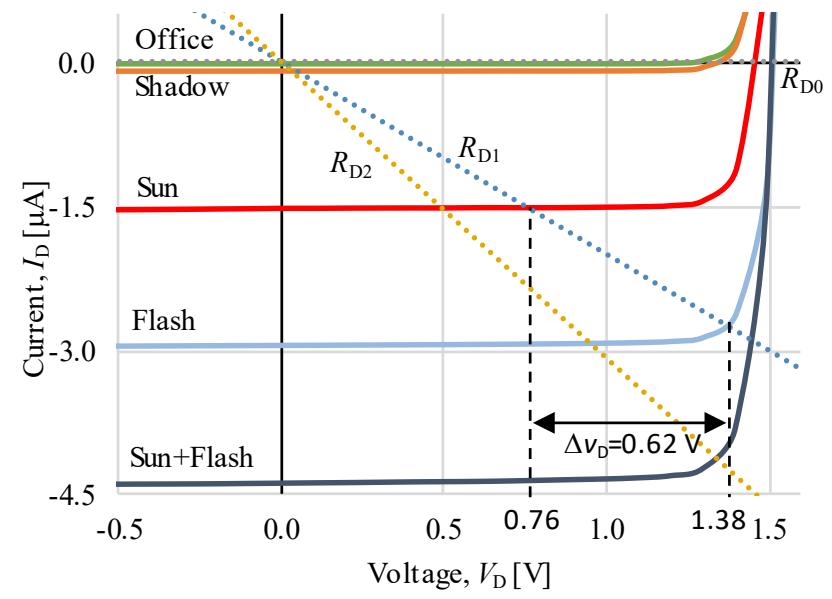

Fig. 2. I/V curves of the LED under test.

TABLE I

VALUES OF $V_{\mathrm{D}}$ AND $\Delta V_{\mathrm{D}}$

\begin{tabular}{ccccccc}
\hline \hline \multirow{2}{*}{ Light } & \multicolumn{2}{c}{$R_{\mathrm{D} 0}$} & \multicolumn{2}{c}{$R_{\mathrm{D} 1}$} & \multicolumn{2}{c}{$R_{\mathrm{D} 2}$} \\
\cline { 2 - 7 } & $v_{\mathrm{D}}$ & $\Delta v_{\mathrm{D}}$ & $v_{\mathrm{D}}$ & $\Delta v_{\mathrm{D}}$ & $v_{\mathrm{D}}$ & $\Delta v_{\mathrm{D}}$ \\
\hline Office & 1.17 & 0.35 & 0.002 & 1.38 & 0.001 & 1.00 \\
Shadow & 1.37 & 0.15 & 0.045 & 1.34 & 0.035 & 0.96 \\
Sun & 1.46 & 0.06 & 0.76 & 0.62 & 0.52 & 0.48 \\
Flash & 1.52 & - & 1.38 & - & 1.00 & - \\
Sun+Flash & 1.52 & 0.06 & 1.45 & 0.69 & 1.37 & 0.85 \\
\hline \hline
\end{tabular}




\section{B. Dynamic Response}

A flashlight with $f_{\mathrm{s}}=25 \mathrm{~Hz}$, which is around the upper limit that can be achieved with the smartphone flashlight, and a duty cycle of $50 \%$ was generated with the emulated flashlight. Table II shows the value of the remaining circuit parameters in Fig. 1 as well as the calculated cut-off frequencies of the BPF. Frequency $f_{2}$ is calculated from (3). Values of the HPF ( $R_{\mathrm{H}}$ and $C_{\mathrm{H}}$ ) are chosen to accomplish (4) and also $R_{\mathrm{H}}>>R_{\mathrm{D}}$ and $C_{\mathrm{D}}, C_{\mathrm{M}}<<C_{\mathrm{H}}$. Then, $f_{1}$ is calculated using (2).

TABLE II

PARAMETERS OF THE WAKE-UP CIRCUIT AND THE CORRESPONDING CUT-OFF FREQUENCIES

\begin{tabular}{ccccccccc}
\hline \hline $\begin{array}{c}R_{\mathrm{D}} \\
(\mathrm{k} \Omega)\end{array}$ & $\begin{array}{c}C_{\mathrm{D}}{ }^{\mathrm{a}} \\
(\mathrm{pF})\end{array}$ & $\begin{array}{c}C_{\mathrm{M}} \\
(\mathrm{pF})\end{array}$ & $\begin{array}{c}f_{2} \\
(\mathrm{kHz})\end{array}$ & $\begin{array}{c}R_{\mathrm{H}} \\
(\mathrm{M} \Omega)\end{array}$ & $\begin{array}{c}C_{\mathrm{H}} \\
(\mathrm{pF})\end{array}$ & $\begin{array}{c}f_{1} \\
(\mathrm{~Hz})\end{array}$ & $\begin{array}{c}R_{\mathrm{M}} \\
(\mathrm{k} \Omega)\end{array}$ & $\begin{array}{c}V_{\mathrm{CC}} \\
(\mathrm{V})\end{array}$ \\
\hline 510 & 16 & 25 & 7.61 & 5.6 & 680 & 41.8 & 560 & 3 \\
\hline \hline
\end{tabular}

${ }^{a}$ Measured with an impedance analyzer (E4990A, Keysight).

Tests were performed outdoors with direct sunlight hitting laterally the LED, apart from the emulated flashlight. Fig. 3 shows the results acquired with an oscilloscope. As can be seen, $v_{\mathrm{D}}$ is a square wave switching from around 500-600 mV when only the sunlight was present to 1.4-1.5 $\mathrm{V}$ when the flashlight also illuminated the LED, which is coherent with the values of $v_{\mathrm{D}}$ shown in Table I for $R_{\mathrm{D} 1}$ (change from Sun to Sun+Flash). A lower voltage with sunlight was now achieved, probably due to a lower intensity of the sunlight than with the case of the static characterization. The effect of the HPF is observed in $v_{\mathrm{H}}$, where peak voltages $\left(\Delta v_{\mathrm{D}}\right)$ around $800-900 \mathrm{mV}$ were generated, enough to activate the NMOS of the VLT stage and make toggle $v_{\mathrm{M}}$ from $3 \mathrm{~V}$ (' 1 ') to $0 \mathrm{~V}$ (' 0 '). Once $v_{\mathrm{H}}$ decreases down $V_{\mathrm{T}}$, around $400 \mathrm{mV}, v_{\mathrm{M}}$ toggles again to a ' 1 ' until the next rising edge of $v_{D}$. This emulated situation is a worst-case scenario since the intensity of the interfering light, sunlight, is high. Even so, the system still works as pretended. DC current consumption of the circuit when not switching was around $0.5 \mathrm{nA}$.

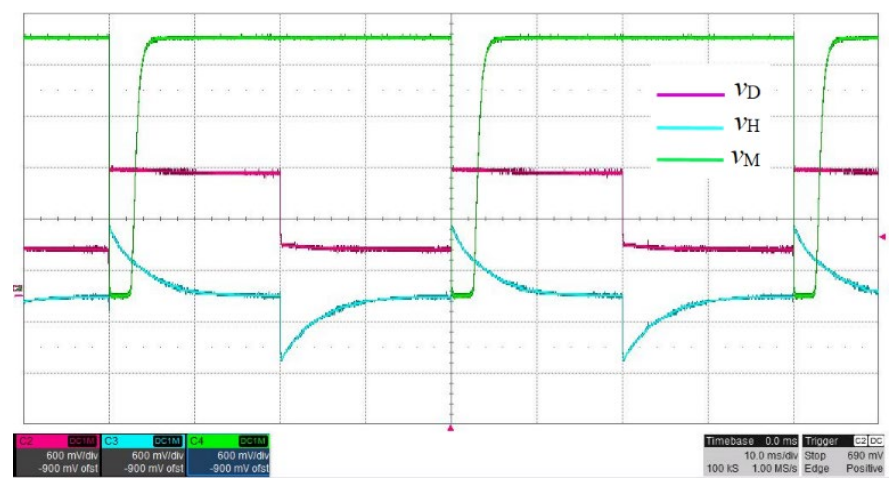

Fig. 3. Dynamic response waveforms of $v_{\mathrm{D}}, v_{\mathrm{H}}$, and $v_{\mathrm{M}}$.

\section{Smartphone Test}

A test was performed outdoors in a sunny day with the smartphone flashlight and the MCU executing the FAC. Fig. 4 shows the resulting waveforms. Pulses are not perfectly periodic but they slightly vary from pulse to pulse; this is a limitation of the Android Operating System since it is not possible to keep accurate track on short times [13]. The waveforms are still similar to those in Fig. 3 with a main difference: the voltage of $v_{\mathrm{D}}$ corresponding to the interfering light is now much lower, around $100-200 \mathrm{mV}$ (top voltage is still $1.4 \mathrm{~V}-1.5$ $\mathrm{V})$, because the smartphone, placed above the LED at a distance around $1 \mathrm{~cm}$, blocks the sunlight, reducing the interfering light to much lower levels, e.g. comparable to Office or Shadow lights. This will be the preferred situation since moving away the smartphone reduces the effect of the flashlight and can increase the interfering light. Again, the HPF blocks the effect of the interfering light and the characteristic waveform can be seen in $v_{\mathrm{H}}$, with a higher peak voltage than before, around 1.3-1.4 V, which is coherent with the values of $\Delta v_{\mathrm{D}}$ shown in Table I for $R_{\mathrm{D} 1}$ and Office and Shadow cases. The VLT stage also generates a digital signal for $v_{\mathrm{M}}$. As a proof of concept, the FAC of the MCU was set to activate a digital output $\left(v_{\mathrm{P}}\right)$ when just three rising edges of $v_{\mathrm{M}}$ were detected within a $300 \mathrm{~ms}$ time interval. Obviously, a more advanced FAC could be used to increase the system security.

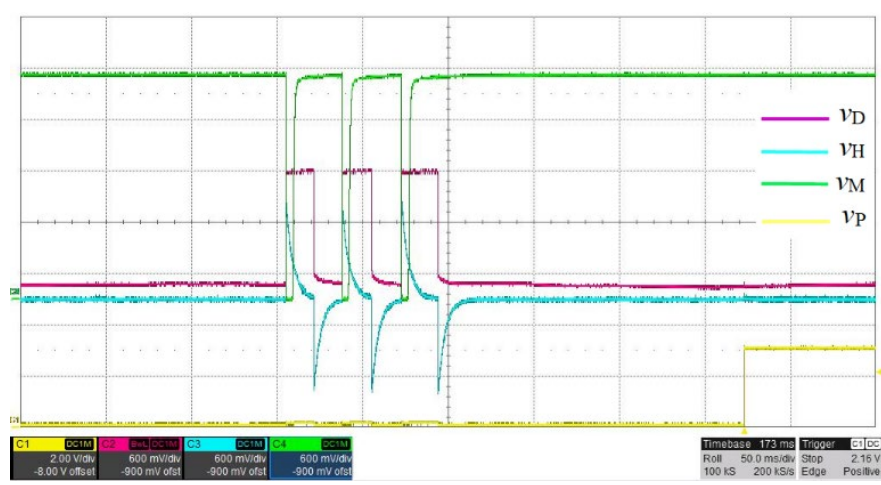

Fig. 4. Waveforms of $v_{\mathrm{D}}, v_{\mathrm{H}}$, and $v_{\mathrm{M}}$ with the smartphone test.

\section{REFERENCES}

[1] R. Yan, H. Sun, and Y. Qian, "Energy-Aware Sensor Node Design With Its Application in Wireless Sensor Networks," IEEE Trans. Instrum. Meas., vol. 62, no. 5, pp. 1183-1191, May 2013.

[2] C. Alippi, G. Anastasi, M. Di Francesco, and M. Roveri, "An Adaptive Sampling Algorithm for Effective Energy Management in Wireless Sensor Networks With Energy-Hungry Sensors," IEEE Trans. Instrum. Meas., vol. 59, no. 2, pp. 335-344, Feb. 2010.

[3] E. Sifuentes, R. Gonzalez-Landaeta, J. Cota-Ruiz, and F. Reverter, "Seat Occupancy Detection Based on a Low-Power Microcontroller and a Single FSR," Sensors, vol. 19, no. 3, p. 699, Feb. 2019.

[4] E. Sifuentes, O. Casas, and R. Pallas-Areny, "Wireless Magnetic Sensor Node for Vehicle Detection With Optical Wake-Up," IEEE Sens. J., vol. 11, no. 8, pp. 1669-1676, Aug. 2011.

[5] E. Ripoll-Vercellone, V. Ferrandiz, and M. Gasulla, "An Add-On Electronic Device to Upgrade Mechanical Gas Meters into Electronic Ones," Proceedings, vol. 2, no. 13, p. 1094, Dec. 2018.

[6] V. Jelicic, M. Magno, D. Brunelli, V. Bilas, and L. Benini, "Benefits of Wake-Up Radio in Energy-Efficient Multimodal Surveillance Wireless Sensor Network," IEEE Sens. J., vol. 14, no. 9, pp. 3210-3220, Sep. 2014.

[7] T. Hakkinen and J. Vanhala, "Ultra-low power wake-up circuit for shortrange wireless communication," in Proc. IET IE, Seattle, WA, USA 2008, pp. 1-4.

[8] W. Lim, T. Jang, I. Lee, H.S. Kim, D. Sylvester, and D. Blaauw, "A $380 \mathrm{pW}$ dual mode optical wake-up receiver with ambient noise cancellation," in Proc. IEEE VLSI-Circuits, Honolulu, HI, USA, 2016, pp. 1-2.

[9] R. Shreyas, "Solving CMOS Transition Rate Issues Using Schmitt Triggers," Texas Instruments, Dallas, TX, USA, White paper SLLA364A, 2017.

[10] M. Kowalczyk and J. Siuzdak, "Photo-reception properties of common LEDs," Opto-Electronics Rev., vol. 25, no. 3, pp. 222-228, Sep. 2017.

[11] E. Ripoll-Vercellone, V. Ferrandiz, J. Aubert, and M. Gasulla, "Using LEDs for Visible Light Communication and as a Wake-up Mechanism in the Internet of Things," in Proc. SenSys, Delft, The Netherlands, 2017, art. no. 49 , pp. 1-2.

[12] E. Ripoll-Vercellone, F. Reverter, V. Ferrandiz, and M. Gasulla, "Experimental characterization of off-the-shelf LEDs as photodetectors for waking up microcontrollers," in Proc. IEEE I2MTC, Auckland, New Zealand, 2019, pp. 1-6.

[13] A. Duquel, R. Stanica, H. Rivano, and A. Desportes, "Decoding methods in LED-to-smartphone bidirectional communication for the IoT," in Proc. GLC, Paris, France, 2018, pp. 1-6. 